
social networks

\section{COMPLEX NETWORKS}

SOCIAL EVOLUTION

COEVOLUTION

Received

15 May 2014

Accepted

11 June 2014

Published

30 June 2014

Correspondence and requests for materials should be addressed to K.L. (lk5622355@pku. edu.cn) or L.W. (longwang@pku.edu. $\mathrm{cn})$

\author{
Kun Li ${ }^{1}$, Rui Cong ${ }^{2}$, Te Wu ${ }^{2}$ \& Long Wang ${ }^{1}$
}

${ }^{1}$ Center for Systems and Control, State Key Laboratory for Turbulence and Complex Systems, College of Engineering, Peking University, Beijing 100871, China, ${ }^{2}$ School of Mechano-Electronic Engineering, Xidian University, Xi'an 710071, China.

The overconfidence, a well-established bias, in fact leads to unrealistic expectations or faulty assessment. So it remains puzzling why such psychology of self-deception is stabilized in human society. To investigate this problem, we draw lessons from evolutionary game theory which provides a theoretical framework to address the subtleties of cooperation among selfish individuals. Here we propose a spatial resource competition model showing that, counter-intuitively, moderate values rather than large values of resource-to-cost ratio boost overconfidence level most effectively. In contrast to theoretical results in infinite well-mixed populations, network plays a role both as a "catalyst" and a "depressant" in the spreading of overconfidence, especially when resource-to-cost ratio is in a certain range. Moreover, when bluffing is taken into consideration, overconfidence evolves to a higher level to counteract its detrimental effect, which may well explain the prosperity of this "erroneous" psychology.

$s$ is well known, confidence leads to success in a wide range of domains including business, job performance, and competitions for champions ${ }^{1-3}$. Ironically, when confidence is relatively high, the whole human beings fall victim to overconfidence, which is considered to be the most "pervasive and potentially catastrophic" of all the cognitive biases by some psychologists ${ }^{4,5}$. Such exaggerated confidence is often blamed for labor strikes, wars, and stock market bubbles ${ }^{6-8}$. But like a coin has two sides, some other authors hold completely different opinions, insisting on its advantages in boosting morale, ambition, resolve and persistence so as to increase the probability of success ${ }^{9-12}$. Regardless of different views, the prevalence of overconfidence is an undeniable fact. For example, by asking people how confident they are of specific beliefs, researchers find that confidence systematically exceeds accuracy, implying people are more sure that they are correct than they deserve to be ${ }^{13,14}$. Further observations also show that individuals incline to form overly positive judgment of their abilities, which is called "better-than-average effect"15.

Overconfidence is a fascinating area of research, since it touches upon many different disciplines ranging from sociology to economics. Mounting effort have been devoted to unveiling the reasons for the pervasiveness of this faulty psychology. Prior work have mostly offered three explanations. The first posits a motivated bias: Individuals are driven to be overconfident because it provides them with psychological benefits ${ }^{16}$. The second explanation highlights the cognitive processes that may sometimes produce directional biases ${ }^{17}$. An additional possibility is that overconfidence enhances the person's social status ${ }^{18}$. Apart from the above results, Johnson and Fowler have presented an evolution model, demonstrating that overconfidence often prevails over accurate assessment as long as benefits from contested resources are sufficiently large compared with the cost of competition $^{12}$. In fact, Johnson and his coauthors used standard assumptions about evolution dynamics ${ }^{19}$, under which whoever get larger fitness are more likely to survive, and better strategies will be imitated.

Treating whether to be overconfident or not as a strategy, we can associate the study of this psychology with evolution game theory which was originally introduced as a tool for studying animal behavior ${ }^{20,21}$, but has become a general approach that transcends almost every aspect of evolutionary biology $\mathrm{y}^{22}$. The traditional approach of evolutionary game theory uses deterministic dynamics to describe infinitely large, well-mixed populations ${ }^{23,24}$. To understand evolutionary game dynamics in finite-sized populations, a stochastic approach is developed ${ }^{25-28}$. Particularly, network has been found to be an important ingredient affecting evolutionary game dynamics. While pioneering works mainly focus on spatial games on regular lattices ${ }^{27,29-37}$, more studies have been expanded to general structured populations, such as small-world ${ }^{38-42}$, scale-free networks ${ }^{43-47}$, and random networks $s^{48,49}$. Taking into account the conspicuous effect of population structure in evolutionary dynamics, one may then ask how psychologies or emotions transmit between interacting individuals who site on specific networks, and whether the transmission has profound significance. In fact, by reconsidering the concept of imitation, Szolnoki et al. find that copying emotions such as goodwill and envy instead of pure strategies from more 
successful players can even resolve social dilemmas on structured populations, additionally pointing out that the emotional profile depends not only on the payoffs but also on the heterogeneity of the interaction network ${ }^{50,51}$.

Inspired by all the previous works above, we propose a spatial resource competition model, simple but general, aiming at dealing with the puzzle how overconfidence spreads and fixes in structured populations. Square lattice prescribes that each individual competes with his 4 nearest neighbors to collect available resources. Then players are more likely to learn the psychology of the successful, despite their real capabilities. Different from the model in $\operatorname{Ref}^{12}$, bluffing behavior has been incorporated into our model. Although bluffing is often argued to be unstable in natural selection ${ }^{9,12}$, it is usually not reliably distinguished from the true ability ${ }^{52}$. In particular, successful disguises (bluffing) and real capacity need a much longer time scale to evolve than that of overconfidence, so they are treated as constant indices in our model. We will show in this study that, moderate values of resource-to-cost ratio $r / c$ create a more favorable environment for the evolution of overconfidence in structured population. More importantly, the intricate relationship between bluffing and overconfidence may be a potential driving force for the high level of overconfidence. The simulation and theoretical analysis details are described in the Methods.

\section{Results}

We begin by presenting overconfidence level $f_{O}$ as a function of resource-to-cost ratio $r / c$ while initial fractions of different types of individuals are equal (see Fig. 1(a)). It is shown that moderate values, rather than high values of $r / c$, boost $f_{O}$ effectively in structured populations. Interestingly, regardless of the values of $\alpha$ and $\beta$, the plateau of high overconfidence level sharply drops to a lower platform once $r / c$ exceeds the critical value 2.0. This can be attributed to the fact that considerable amount of resource stimulates everyone to make a claim actively, leading to intensive conflicts that help one to recognize his real capability. Moreover, the existence of bluffing plays a key role on the prosperity of overconfidence, especially when $r / c$ is high. As is shown in Fig. 1(a), without bluffing $(\beta=0), f_{O}$ is stabilized at a much lower level than that when bluffing exists for $r / c>2.0$. Players seem to be more apt to be overconfident when facing opponents who are not as powerful as they display, which accords with common beliefs. However, excessive overconfidence intensity might inhibit its spreading among the population. For instance, when $\alpha \geq 0.5$ one individual overestimates himself so seriously that he can even challenge opponents from higher rank of real capability (the rank gap is 0.5 ), and this choice never pays off.

As a comparison, we also consider the evolution of overconfidence in well-mixed populations (see Fig. 1(b)), by means of extended replicator dynamic (see Methods for details). It is found that network structure works as a "double edged sword": When $r / c$ is relatively small, it plays a role as "catalyst" to impel the spreading of overconfidence; When resource is rich (larger $r / c$ ), it acts as "depressant" to cool down the atmosphere. The primary cause lies in the fact that network produces a local "celebrity effect"53,54. Specifically, individuals with the highest real capability get more payoff in each game round, and thus do not incline to change their overconfidence states. In networks, they act as hubs to influence their neighbors, forming non-overconfidence clusters to resist invasions (detailed explanations will be discussed later). Therefore no matter how large $r / c$ is, the existing unbiased elites ensure that overconfidence cannot dominate the whole population. In contrast, in well-mixed populations individuals always imitate the "fittest" strategy globally, thereby $x^{*}$ is more sensitive to the values of $r / c$ than $f_{O}$ does, and $x^{*}$ will finally reach a fully overconfident state if resource is rich enough $(r / c>4.0)$ when bluffing exists. However, common traits in both structured and unstructured population exists that bluffing promotes overconfidence level (see Fig. 1 (a) and (b)).

We next evaluate the impact of overconfidence intensity $\alpha$ and bluffing intensity $\beta$ on overconfidence level $f_{O}$, as presented in Fig. 2 . When $r / c=1.5$ (see Fig. 2(a)), $f_{O}$ sustains a high level over the whole parameter plane of $\alpha$ and $\beta$. This confirms that moderate values of resource-to-cost ratio elevate overconfidence, which is robust to changes in overconfidence intensity and bluffing intensity. However, when $r / c=3$ (a large resource-to-cost ratio, see Fig. 2(b)), distinction in $f_{O}$ with respect to $\alpha$ and $\beta$ becomes more
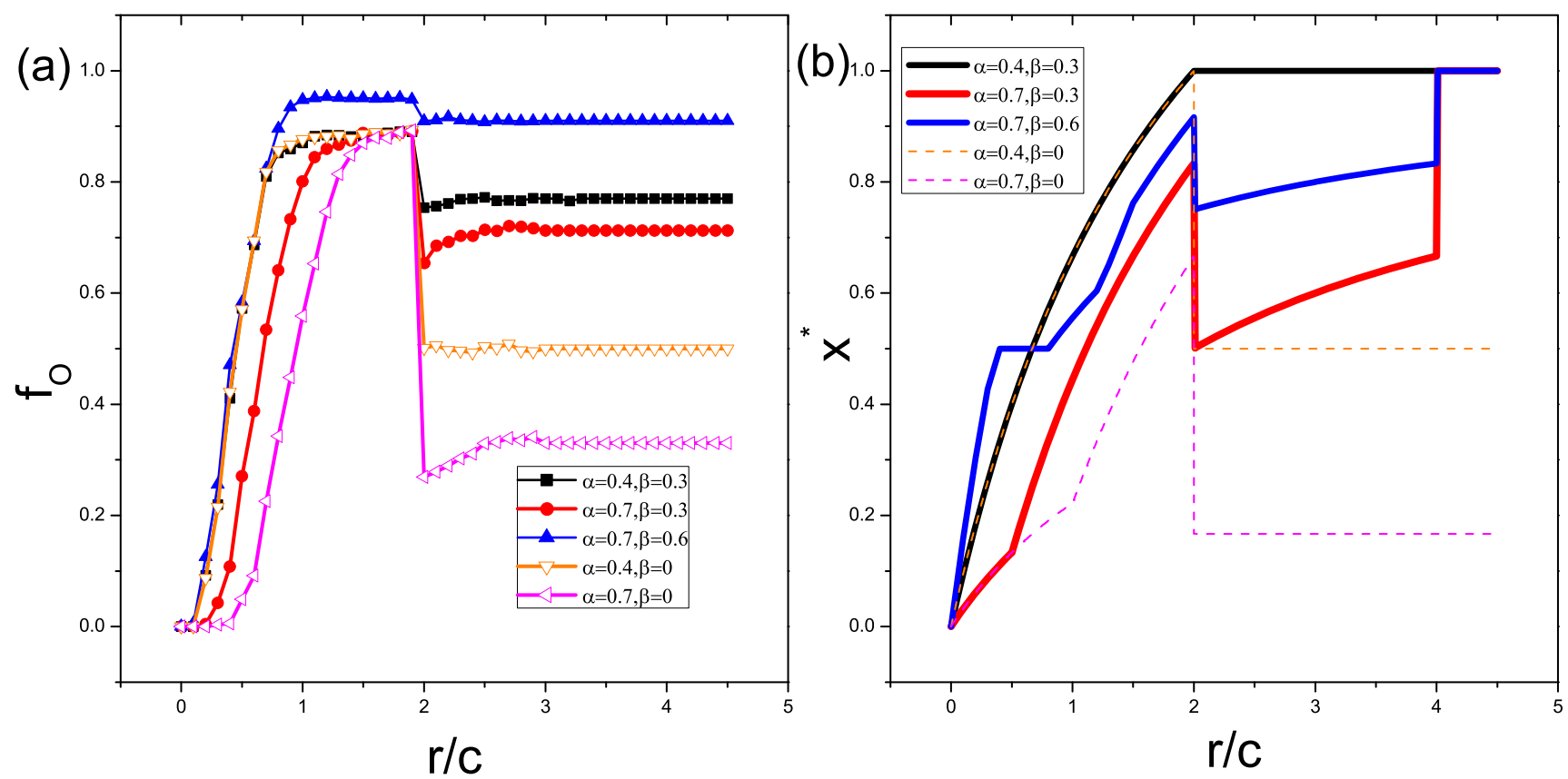

Figure $1 \mid$ (a) Stable fraction of overconfident individuals $f_{O}$ in structured population and (b) equilibrium frequency of overconfident individuals $x^{*}$ in infinite well-mixed population as a function of resource-to-cost ratio $r / c$ for different values of $\alpha$ and $\beta$. Data presented in Panel (a) are obtained by means of Monte Carlo simulations, while in Panel (b) are obtained by means of extended replicator dynamic (see Methods for details). Hollow symbols in Panel (a) and dash line in Panel (b) correspond to cases where bluffing does not exist $(\beta=0)$. Other parameters: $f_{I O}=0.5, f_{B}=0.5$. 

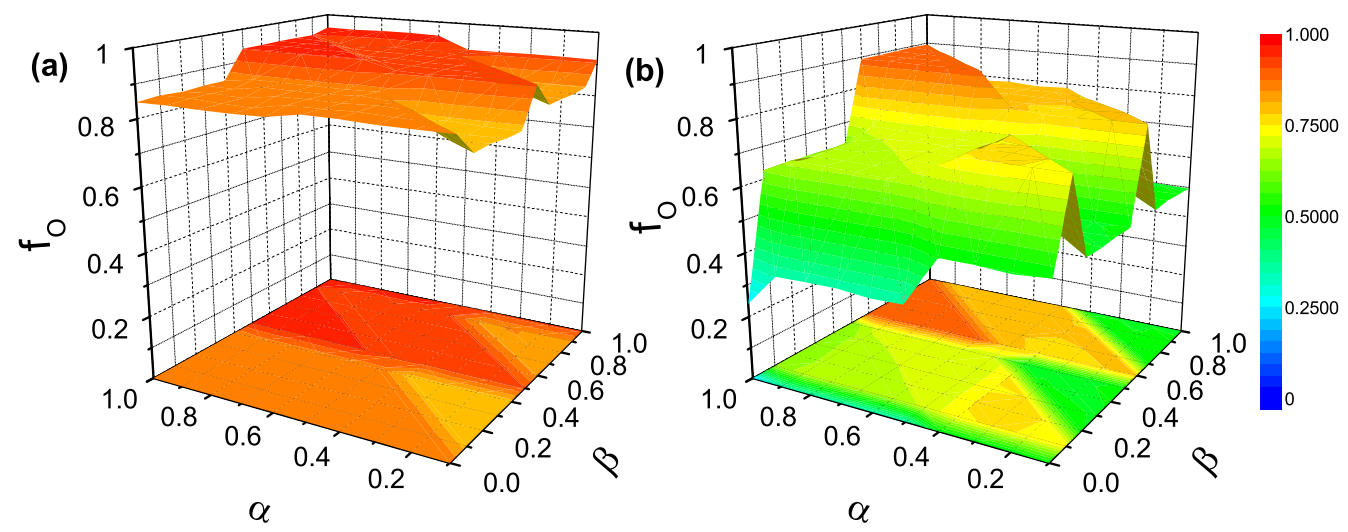

Figure $2 \mid$ The 3-D plots showing the stationary fraction of overconfident individuals $f_{O}$ in dependence on both $\alpha$ and $\beta$ for $(a) r / c=1.5$ and $(b) r / c=3$. The minimum $f_{O}$ is about 0.77 in Panel (a) showing that overconfidence is always favored under moderate values of $r / c$. When $r / c$ is high, $f_{O}$ becomes more strongly dependent on the combination of $\alpha$ and $\beta$. The maximum $f_{O}$ is reached when both $\alpha$ and $\beta$ are high, indicating that higher values of overconfidence is evolved in response to higher bluffing activities.

conspicuous. The maximum $f_{O}$ is reached within the region where large $\alpha$ is accompanied by large $\beta$. Note that for any fixed value of $\beta$, $f_{O}$ is always larger within the region above diagonal (i.e., $\alpha>\beta$ ) than below (i.e., $\alpha<\beta$ ). In other words, an overconfidence intensity slightly larger than bluffing intensity induces higher overconfidence level. In this sense, overconfidence is evolved as a tool to conquer the negative effect of others' bluffing. One should believe himself more than believing others?.

We have further studied the impact of initial frequencies of overconfident individuals $f_{I O}$ and bluffing individuals $f_{B}$ on the final overconfidence level $f_{O}$, as presented in Fig. 3. It can be observed that at any given value of $f_{I O}$, increasing $f_{B}$ will elevate $f_{O}$. A larger $f_{B}$ means that more individuals display an exaggerated capability. When surrounded by more bluffing neighbors, one is more likely to be profitable if he is overconfident. Meanwhile, for any given $f_{B}$, $f_{O}$ also monotonously increases with $f_{I O}$. Unlike the influence of initial frequency on the evolution of cooperation in structured population ${ }^{55}$, we find that the system always converges to a higher overconfidence level compared to the initial one (i.e., $f_{O}>f_{I O}$ ), showing that overconfidence is constantly favored by natural selection once bluffing

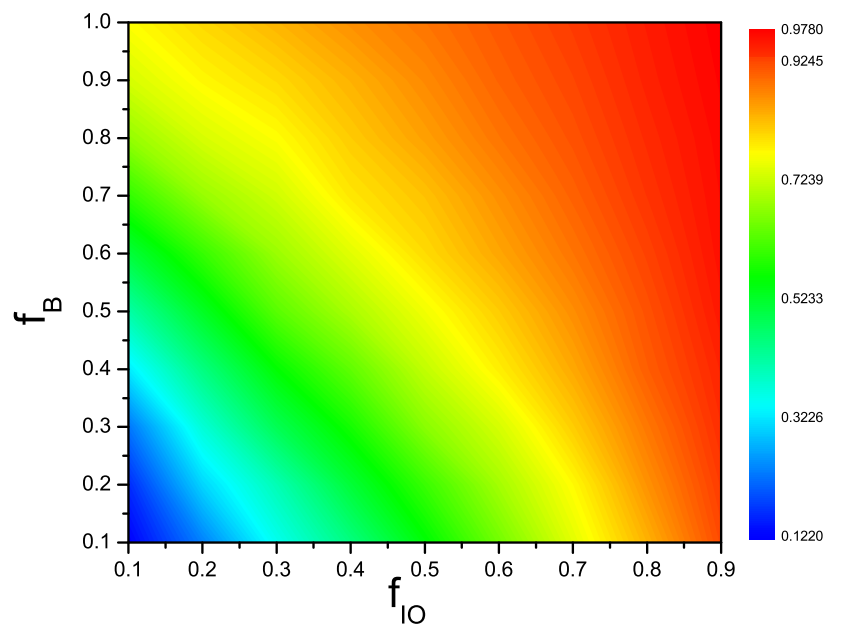

Figure $3 \mid$ Influence of initial overconfidence level $f_{I O}$ and initial bluffing level $f_{B}$ on stationary fraction of overconfident individuals $f_{O}$ in the contour form. Parameters are: $\alpha=0.4, \beta=0.3$, and $r / c=2.5$. Similar results can be obtained whenever $\alpha>\beta$ and $r / c>2.0$. Overall, larger $f_{I O}$ corresponds to higher $f_{O}$. For any given value of $f_{I O}$, increasing $f_{B}$ further promotes $f_{O}$, showing that overconfidence is favored when bluffing exists. exists. Our investigation shows that the prosperity of bluffing stimulates overconfidence to prevail, given that $\alpha>\beta$ and $r / c>2.0$.

For better understanding the influence of network structure on the evolution of overconfidence, we present in Fig. 4 some typical snapshots of strategy patterns in time evolution. It can be observed that, regardless of the different initial distributions, overconfidence spreads quickly and flourishes. Individuals who have high real ability $(\mathrm{H})$, considered as "elites" here, play decisive roles in guiding social mentality, and thus they become the core of the clusters. As evolution proceeds, the number of overconfident elites (blue) increases, indicating that they have an advantage over the unbiased elites (yellow). However, the latter can also take advantage of the network structure to form some solid clusters (yellow + red) to resist invasion, which explains why the unbiased ones will not die out in the long run in structured populations. Notably, the overconfidence clusters (blue + green) tend to connect with each other, forming larger ones to expand their territory, especially when initial fraction of overconfidence is relatively high $\left(f_{I O}=0.3\right)$. Note that besides network reciprocity ${ }^{29,56,57}$, local "celebrity effect" also plays important roles. Individuals are susceptible to neighbor elites' psychology. These followers of overconfident or unbiased elites work as "buffer" in between, preventing the direct confrontation of two types of elites.

Moreover, who benefit more from such a resource competition game draws our attention, and thus we compare the average payoffs of different types of individuals in Fig. 5. Firstly, real ability is fatal in deciding one's faith in resource competition. The higher one's real ability is, the higher average payoff one acquires. Just as illustrated in Fig. 5 (a), there exist clear-cut gaps between income hierarchies, and the lower level can never outcompete the upper one, no matter how the initial frequencies of bluffing and overconfident individuals are. Secondly, whether bluffing ones perform much better than unbiased ones also depends on their real abilities. The low capability bluffing ones have the whip hand over those who do not bluff, especially when bluffing and overconfidence are rare initially (see the left panel of Fig. 5(b)). While for middle and high ability, the advantage of bluffing is relatively low (see the middle and right panel of Fig. 5 (b)). Common for individuals of any level of real ability, bluffing is more effective when the others do not bluff and meanwhile do not develop the overconfidence, the useful tool countervailing bluffing.

Furthermore, it is worth studying the situation not only overconfidence state (OS), but also bluffing state (BS) could be subject to evolution process. In other words, we consider an extended model that individuals can mimic any feature of others except the real ability. Different from the case with fixed bluffing level (see Fig. 1), where $f_{O}$ suddenly drops once $r / c>2.0$, here $f_{O}$ monotonously increases with $r / c$ (see Fig. 6). And in the latter case, $f_{O}$ is higher than 

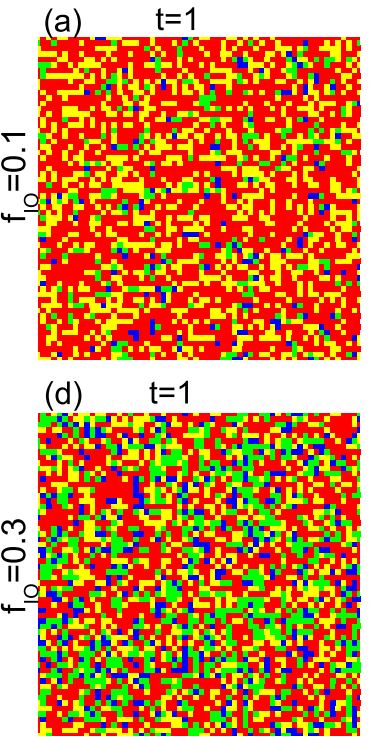
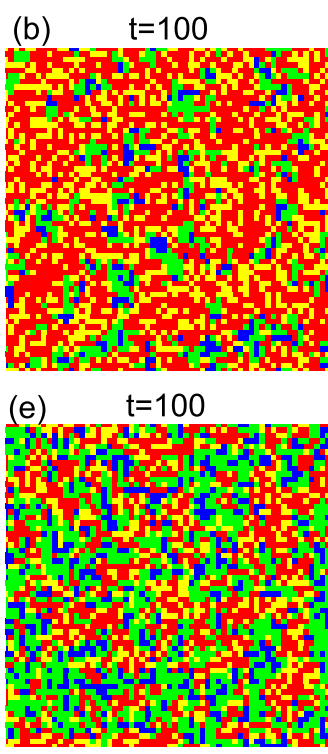
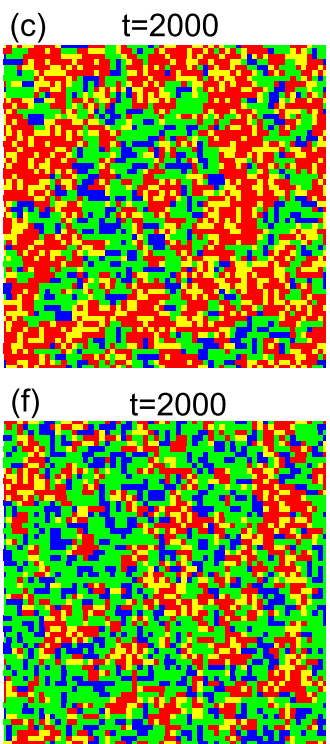

Figure $4 \mid$ Typical snapshots of spatial patterns formed by different types of individuals under initial overconfidence levels $f_{I O}=0.1((a)-(c))$ and $f_{I O}=0.3((\mathrm{~d})-(\mathrm{f}))$ at different time steps. Overconfident ones are colored blue (high real ability) and green (middle and low real ability). Unbiased ones are colored yellow (high real ability) and red (middle and low real ability). The stationary fractions of overconfident ones are $42.5 \%$ for $f_{I O}=0.1$ and $62.6 \%$ for $f_{I O}=0.3$. In structured population, high real ability but unbiased individuals form clusters to resist the invasion by overconfident ones, explaining the survival of unbiased ones even with high $r / c$. The size of the square lattices is $100 \times 100$. Other parameters: $\alpha=0.4, \beta=0.3$, and $r / c=3$.

the former. Meanwhile, $f_{B}$ evolves to a higher value relative to the initial one (0.5) when $r / c>2$. And $f_{B}$ is further promoted with larger bluffing intensity $\beta$ (see Fig. 6(b)). This may provide an explanation why $f_{O}$ evolves to a higher level and does not decrease when $r / c>2$. Just like what we have shown in the scenario with fixed $f_{B}$ (see Fig. 3), a larger percentage of bluffing individuals induces higher overconfidence level. It seems that overconfidence may gain more popularity if the skill of bluffing can be learned easily.

\section{Discussion}

In summary, we have investigated the impact of spatial structure and bluffing on the evolution of overconfidence in the resource competition game. In contrast to previous results in well-mixed populations ${ }^{12}$, the stable state is no longer composed of monomorphic overconfident individuals when the resource-to-cost ratio $r / c$ is sufficiently large. Overconfidence never dominates the whole population and is more adaptive for moderate $r / c$. This is partially ascribed to the local celebrity effect caused by the population structure and real ability hierarchies. Furthermore, a potential benefit far exceeding the cost of competition stimulates more conflicts ${ }^{12,58}$, which in turn depresses the psychology of self-deception, i.e., the overconfidence.

The existence of bluffing behavior usually leads to ambiguity in one's perception about other's real ability. Our study shows that bluffing promotes overconfidence. Overconfidence is beneficial for individuals in an environment where bluffing is prevalent, because it makes them dare claim and compete for the resource, which would not happen without such "erroneous self-deception". Therefore overconfidence is just like a tool to conquer the negative effect of bluffing. This conclusion is robust to initial distributions or intensity of overconfidence and bluffing. Furthermore, the advantage of bluffing is more significant within communities of relatively low real abilities. If bluffing can be learned by others, it will become popular among individuals, further promoting overconfidence level.

All in all, the spreading of overconfidence is investigated in a more realistic model, and we propose that the existence of bluffing is the key factor for the prosperity of the bias towards exaggerated personal qualities and capabilities. We hope that these observations could
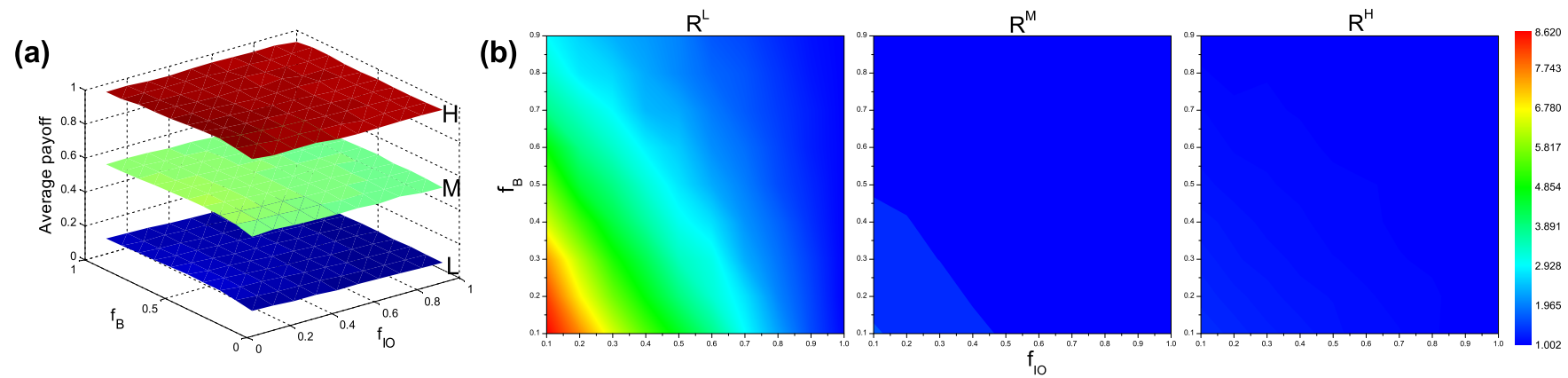

Figure 5 Comparison of the payoffs of individuals with different real abilities. (a) The 3-D plot showing the normalized average accumulated payoffs of individuals with high $\left(\mathrm{H}\right.$, crimson), middle $\left(\mathrm{M}\right.$, light green), and low (L, blue) real abilities respectively, as a function of $f_{I O}$ and $f_{B}$. H ranges from 0.9350 to 1; $\mathrm{M}$ ranges from 0.4859 to 0.5521 ; L ranges from 0.0443 to 0.1065 . (b) Average payoff ratio between the individuals who bluff and those who do not $\left(R=\right.$ Payof $f_{\text {bluff } f} /$ Payof $\left.f_{\text {non-bluff }}\right)$ as a function of $f_{I O}$ and $f_{B}$, when their real abilities are low $\left(R^{L}\right)$, middle $\left(R^{M}\right)$, and high $\left(R^{H}\right)$ respectively. $R^{L}$ ranges from 1.03 to $8.62 ; R^{M}$ ranges from 1.01 to $1.634 ; R^{H}$ ranges from 1.002 to 1.322 . Data point is obtained by averaging over a period of 10000 time steps, and we have checked that longer time period does not qualitatively change the results. Other parameters: $\alpha=0.4, \beta=0.3$, and $r / c=3$. 

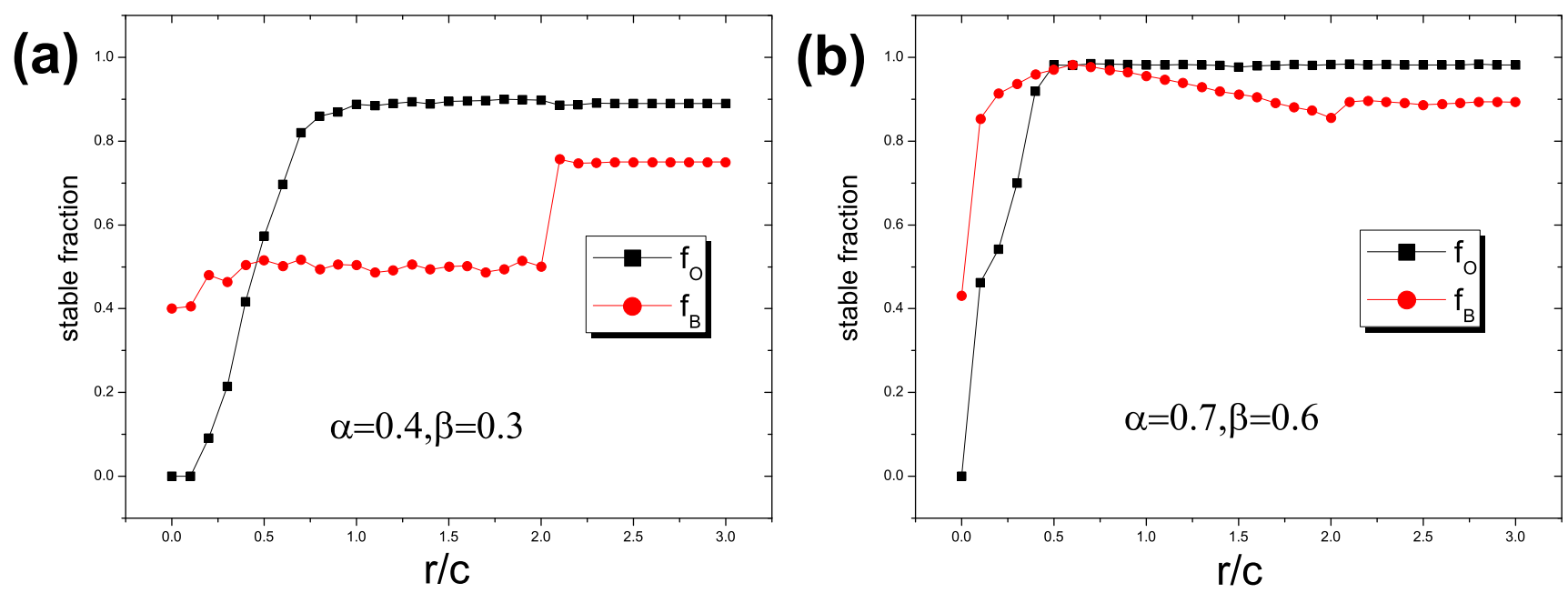

Figure $6 \mid$ Stable fractions of overconfident individuals $f_{O}$ and bluffing individuals $f_{B}$ as a function of $r / c$ for different values of $\alpha$ and $\beta$, when players can learn both the overconfidence state and the bluffing state of others. In Panel (a), $\alpha=0.4$ and $\beta=0.3$. Bluffing prevails once $r / c$ exceeds the critical value 2.0. Overconfidence does not drop to lower level with increasing $r / c$ and remains above 0.89. In Panel (b), $\alpha=0.7$ and $\beta=0.6$. Bluffing almost dominate the whole range of $r / c$. Overconfidence stays above 0.98 for $r / c>0.5$. Other parameters: $f_{I O}=0.5$, and $f_{I B}=0.5$.

provide some insight into understanding this widespread erroneous psychology in human society.

\section{Methods}

Mathematical model. Within this work, the basic model is a resource competition game (RCG). Without loss of generality, an individual $i$ in our model is characterized by real capability $\theta_{i}$, overconfidence intensity $\alpha$, and bluffing intensity $\beta$, where $\theta_{i} \in$ $\Theta=\{1,1.5,2\}$, corresponding to low $(\mathrm{L})$, middle $(\mathrm{M})$, and high $(\mathrm{H})$ real capabilities, respectively. The value of $\alpha$ represents the perception error of a player about his own ability when he is overconfident, while $\beta$ represents how much stronger a player displays than he really is. Whoever dare compete for resource is at least confident, so we assume no self-abasement in this model. In a RCG, $i$ belives he owns a "selfperceived capability" $k_{i}$ as:

$$
k_{i}=\theta_{i}+u_{i} \alpha
$$

where $u_{i}=1$ if $i$ is overconfident and 0 otherwise. He also exhibits his "displaying capability" $m_{i}$ as:

$$
m_{i}=\theta_{i}+v_{i} \beta
$$

where $v_{i}=1$ if $i$ bluffs and 0 otherwise. Supposing a resource $r$ is potentially available to individuals that claim it, and two players $i$ and $j$ both can claim this resource, a RCG takes place. If neither individual claims the resource, then no fitness is gained. If only one individual makes a claim, then it acquires the resource and gains fitness $r$ and the other individual gains nothing. If both individuals claim the resource, then each individual pays a cost $c$ due to the conflict between them, and the results depend on their real capabilities. Therein player $i$ faced with $j$ gains a payoff $P_{i j}$, which can be calculated as:

- (1) If $k_{i}>m_{j}$ and $k_{j}<m_{i}, i$ claims but $j$ does not, and thus $P_{i j}=r$.

- (2) If $k_{i}<m_{j}, i$ will not claim, and $P_{i j}=0$.

- (3) If $k_{i}>m_{j}$ and $k_{j}>m_{i}$, a conflict happens between i and j, whose real capabilities determine what they can get: If $\theta_{i}>\theta_{j}, P_{i j}=r-c$; If $\theta_{i}<\theta_{j}$, $P_{i j}=-c$; If $\theta_{i}=\theta_{j}, P_{i j}=r / 2-c$, i.e., $r$ is equally distributed between them after a battle.

- (4) If $k_{i}=m_{j}$, the relation between $r$ and $c$ should be considered: If $r \leq 2 c, i$ will not claim because the upcoming conflict promises no positive benefit, and thus $P_{i j}=$ 0 ; If $r>2 c$ and $k_{j}<m_{i}$, $i$ gains the whole resource without any payout, and $P_{i j}=r$; If $r>2 c$ and $k_{j} \geq m_{i}$, the clash is inevitable, and therefore $P_{i j}$ can be calculated as in case (3).

Players are located on the vertices of $L \times L$ square lattices with periodic boundary conditions. Capability rank and bluffing state (BS) are considered to be natural instincts, and thus are constant throughout the evolution. Individuals can only imitate overconfidence states (OS) of others. In an extended model we also explore the case where individuals can mimic both OS and BS of others. $f_{I B}$ denotes the fraction of individuals that bluff. Monte Carlo simulations of the game are carried out comprising two elementary steps. Firstly, each player $i$ collects its payoff $P_{i}$ by interacting with its four nearest neighbors through RCG. The payoff of player $i$ can be calculated as:

$$
P_{i}=\sum_{j \in \Omega_{(i)}} P_{i j}
$$

where $\Omega_{(i)}$ represents players in $i$ 's neighborhood. Secondly, players consider chan ging their OS synchronously. Specifically, player $i$ adopts the OS of the randomly selected neighbor $j$ with the probability

$$
T\left(P_{j}-P_{i}\right)=\frac{1}{1+\exp \left[\left(P_{i}-P_{j}\right) / K\right]}
$$

where $K$ characterizes the level of uncertainty in strategy adoption ${ }^{59}$. Without loss of generality we use $K=0.1$, and we have confirmed that different values of $K$ do not qualitatively change the simulation results.

Extended replicator dynamic. For comparison, evolutionary dynamics of overconfidence in well-mixed population is also investigated. In replicator dynamics, a differential equation governs the evolution of the densities of different strategies, lending to the notion of evolutionary stability ${ }^{23,60,61}$. Further, enlightened by $\operatorname{Re}^{62}$, we can consider the whole population to be specially community-structured ${ }^{63-68}$, depending on different character profiles of individuals. There are 6 character profiles, specified by a 2-bit feature name $\left[a_{1} a_{2}\right]$ where $a_{1} \in[L, M, H]$ indicates the capability level of this individual is low, middle, or high, while $a_{2} \in[B, N]$ indicates whether he bluffs or not, indeed forming 6 communities. Players imitate overconfidence states of others but cannot change their characters, and thus the size of each community is constant and the members cannot migrate among communities. Strategies A (or B) indicates that an individual is overconfident (or not), while $x_{i}$ and $y_{i}(i=L B, L N, M B, M N, H B$, $H N$ ) denote the fractions of individuals adopting strategy A and B in community $i$ respectively. Here $x_{i}+y_{i}=1$. We use $\rho_{i}$ to denote the fraction of community $i$ members in the whole population, and then $\sum_{i} \rho_{i}=1$. Hence any individual interacts with a player from community $i$ with probability $\rho_{i}$ according to the well-mixed assumption. In each community, the standard replicator equation takes the form of:

$$
\left\{\begin{array}{l}
\dot{x}_{i}=x_{i}\left(\eta_{A i}-\bar{\eta}_{i}\right) \\
\dot{y}_{i}=y_{i}\left(\eta_{B i}-\bar{\eta}_{i}\right)
\end{array} \quad(i=L B, L N, M B, M N, H B, H N)\right.
$$

where $\eta_{A i}$ and $\eta_{B i}$ are the fitness of overconfident and unbiased people in community $i$ respectively, and $\bar{\eta}_{i}=x_{i} \eta_{A i}+y_{i} \eta_{B i}$ is the average fitness of individuals in community $i$. By solving above equations, we can obtain $x_{i}^{*}$, the stable fixed state of overconfidence (SFSO) of community $i$. Hence we can derive the SFSO of the entire system as

$$
x^{*}=\sum_{i} \rho_{i} x_{i}^{*}
$$

Assume that individuals are randomly distributed among the communities, i.e., for each community $i(i=L B, L N, M B, M N, H B, H N), \rho_{i}=1 / 6$, and that each one has equal probability 0.5 to be overconfident or not. Thus initial overconfidence level for each community is $x_{i}^{(0)}=1 / 12$. Taking $\alpha=0.4$ and $\beta=0.3$ as an example, we can obtain the dynamic equations as: 


$$
\left\{\begin{array}{l}
r \leq 2 c:\left\{\begin{array}{l}
\dot{x}_{L B}+\dot{x}_{L N}=\left(x_{L B}-x_{L B}^{2}+x_{L N}-x_{L N}^{2}\right)\left[\left(-\frac{r}{12}-\frac{c}{6}\right)\left(x_{L B}+x_{L N}\right)+\frac{r}{3}\right] \\
\dot{x}_{M B}+\dot{x}_{M N}=\left(x_{M B}-x_{M B}^{2}+x_{M N}-x_{M N}^{2}\right)\left[\left(-\frac{r}{12}-\frac{c}{6}\right)\left(x_{M B}+x_{M N}\right)+\frac{r}{3}\right] \\
\dot{x}_{H B}+\dot{x}_{H N}=\left(x_{H B}-x_{H B}^{2}+x_{H N}-x_{H N}^{2}\right)\left[\left(-\frac{r}{12}-\frac{c}{6}\right)\left(x_{H B}+x_{H N}\right)+\frac{r}{3}\right]
\end{array}\right. \\
r>2 c: \begin{cases}\dot{x}_{a_{1} B}=x_{a_{1} B}\left(1-x_{a_{1} B}\right)\left[\left(\frac{r}{2}-c\right) * \frac{x_{a_{1} B}}{6}+r * \frac{1-x_{a_{1} B}}{6}\right] \\
\dot{x}_{a_{1} N}=x_{a_{1} N}\left(1-x_{a_{1} N}\right)\left[\left(\frac{r}{2}-c\right) * \frac{1}{6}\right]\end{cases}
\end{array}\right.
$$

The stable equilibrium point of the above differential equations, i.e., the SFSO, is:

$$
x^{*}= \begin{cases}\frac{2 r}{r+2 c} & (r \leq 2 c) \\ 1 & (r>2 c)\end{cases}
$$

Different SFSOs with respect to other overconfidence and bluffing intensities are shown in the main text.

1. Taylor, S. \& Brown, J. Positive illusions and well-being revisited-separating fact from fiction. Psychol. Bull. 116, 21-27 (1994).

2. Eisen, C. Confidence: How winning streaks and losing streaks begin and end. Libr. J. 129, 67 (2004).

3. Dunn, D. S. A primer in positive psychology. J. Soc. Clin. Psychol. 27, 896-898 (2008).

4. Pallier, G. The role of individual differences in the accuracy of confidence judgments. J. Gen. Psychol. 129, 257-299 (2002).

5. Koehler, J. The psychology of judgment and decision-making. Contemp. Psychol. 40, 315-316 (1995).

6. Babcock, L. \& Olson, C. The causes of impasses in labor disputes. Ind. Relat. 31, 348-360 (1992).

7. Kampmark, B. Overconfidence and war: The havoc and glory of positive illusions. J. Am. Stud 40, 178-179 (2006).

8. Aumann, R. Agreeing to disagree. Ann. Stat 4, 1236-1239 (1976).

9. Trivers, R. The elements of a scientific theory of self-deception. Ann. Ny. Acad. Sci 907, 114-131 (2000).

10. McKay, R. T. \& Dennett, D. C. The evolution of misbelief. Behav. Brain. Sci. 32, 493 (2009).

11. West, S. Deceit and self-deception: Fooling yourself the better to fool others. Nature 478, 314-315 (2011).

12. Johnson, D. D. P. \& Fowler, J. H. The evolution of overconfidence. Nature 477, 317-320 (2011).

13. Roediger, H. \& Mcdermott, K. Creating false memories-Remembering words not presented in lists. J. Exp. Psychol. Learn. 21, 803-814 (1995).

14. Henson, R., Rugg, M., Shallice, T. \& Dolan, R. Confidence in recognition memory for words: Dissociating right prefrontal roles in episodic retrieval. J. Cognitive. Neurosci. 12, 913-923 (2000).

15. Alicke, M., Klotz, M., Breitenbecher, D., Yurak, T. \& Vredenburg, D. Personal contact individuation, and the better-than-average effect. J. Pers. Soc. Psychol. 68 , 804-825 (1995).

16. Dunning, D., Leuenberger, A. \& Sherman, D. A new look at motivated inferenceAre self-serving theories of success a product of motivational forces. J. Pers. Soc. Psychol. 69, 58-68 (1995).

17. Kruger, J. \& Dunning, D. Unskilled and unaware of it: How difficulties in recognizing one's own incompetence lead to inflated self-assessments. J. Pers. Soc. Psychol. 77, 1121-1134 (1999).

18. Anderson, C., Brion, S., Moore, D. A. \& Kennedy, J. A. A status-enhancement account of overconfidence. J. Pers. Soc. Psychol. 103, 718-735 (2012).

19. Nowak, M. A. Evolutionary Dynamics: Exploring the Equations of Life (Harvard Univ. Press, 2006).

20. Smith, J. M. \& Price, G. R. The logic of animal conflict. Nature 246, 15-18 (1973).

21. Smith, J. M. Evolution and the Theory of Games (Cambridge Univ. Press, 1982).

22. Nowak, M. A. \& Sigmund, K. Evolutionary dynamics of biological games. Science 303, 793-799 (2004).

23. Hofbauer, J. \& Sigmund, K. Evolutionary Games and Population Dynamics (Cambridge Univ. Press, 1998).

24. Hofbauer, J. \& Sigmund, K. Evolutionary game dynamics. B. Am. Math. Soc. 40, 479-519 (2003).

25. Schaffer, M. Evolutionarily stable strategies for a finite population and a variable contest size. J. Theor. Biol. 132, 469-478 (1988).

26. Traulsen, A. \& Nowak, M. A. Evolution of cooperation by multilevel selection. Proc. Natl. Acad. Sci. USA 103, 10952-10955 (2006).

27. Perc, M. Transition from gaussian to levy distributions of stochastic payoff variations in the spatial prisoner's dilemma game. Phys. Rev. E 75, 022101 (2007).

28. Traulsen, A. \& Nowak, M. A. Chromodynamics of cooperation in finite populations. PLoS ONE 2, e270 (2007).

29. Nowak, M. A. \& May, R. M. Evolutionary games and spatial chaos. Nature 359, 826-829 (1992).
30. Nakamaru, M., Matsuda, H. \& Iwasa, Y. The evolution of cooperation in a latticestructured population. J. Theor. Biol. 184, 65-81 (1997).

31. Szabó, G. \& Töke Evolutionary prisoner's dilemma game on a square lattice. Phys. Rev. E 58, 69-73 (1998).

32. Szabó, G., Antal, T., Szabo, P. \& Droz, M. Spatial evolutionary prisoner's dilemma game with three strategies and external constraints. Phys. Rev. E 62, 1095-1103 (2000).

33. Szolnoki, A., Perc, M. \& Danku, Z. Making new connections towards cooperation in the prisoner's dilemma game. EPL 84, 50007 (2008).

34. Szabó, G. \& Hauert, C. Phase transitions and volunteering in spatial public goods games. Phys. Rev. Lett. 89, 118101 (2002).

35. Szolnoki, A., Wang, Z. \& Perc, M. Wisdom of groups promotes cooperation in evolutionary social dilemmas. Sci. Rep. 2, 576 (2012).

36. Perc, M. Sustainable institutionalized punishment requires elimination of secondorder free-riders. Sci. Rep. 2, 344 (2012).

37. Wang, X., Perc, M., Liu, Y., Chen, X. \& Wang, L. Beyond pairwise strategy updating in the prisoner's dilemma game. Sci. Rep. 2, 740 (2012).

38. Santos, F. C., Rodrigues, J. F. \& Pacheco, J. M. Epidemic spreading and cooperation dynamics on homogeneous small-world networks. Phys. Rev. E 72, 056128 (2005).

39. Ren, J., Wang, W. X. \& Qi, F. Randomness enhances cooperation: A resonancetype phenomenon in evolutionary games. Phys. Rev. E 75, 045101 (2007).

40. Fu, F., Liu, L. H. \& Wang, L. Evolutionary prisoner's dilemma on heterogeneous Newman-Watts small-world network. Eur. Phys. J. B. 56, 367-372 (2007).

41. Perc, M. Double resonance in cooperation induced by noise and network variation for an evolutionary prisoner's dilemma. New J. Phys. 8, 183 (2006).

42. Chen, X. \& Wang, L. Promotion of cooperation induced by appropriate payoff aspirations in a small-world networked game. Phys. Rev. E 77, 017103 (2008).

43. Santos, F. C. \& Pacheco, J. M. Scale-free networks provide a unifying framework for the emergence of cooperation. Phys. Rev. Lett. 95, 098104 (2005).

44. Santos, F. C., Pacheco, J. M. \& Lenaerts, T. Evolutionary dynamics of social dilemmas in structured heterogeneous populations. Proc. Natl. Acad. Sci. USA 103, 3490-3494 (2006).

45. Szolnoki, A., Perc, M. \& Danku, Z. Towards effective payoffs in the prisoners dilemma game on scale-free networks. Physica A 387, 2075-2082 (2008).

46. Perc, M. Evolution of cooperation on scale-free networks subject to error and attack. New J. Phys. 11, 033027 (2009).

47. Wu, T., Fu, F., Zhang, Y. \& Wang, L. Adaptive role switching promotes fairness in networked ultimatum game. Sci. Rep. 3, 1550 (2013).

48. Barabási, A. L. \& Albert, R. Emergence of scaling in random networks. Science 286, 509-512 (1999).

49. Szolnoki, A. \& Perc, M. Emergence of multilevel selection in the prisoner's dilemma game on coevolving random networks. New J. Phys. 11, 093033 (2009).

50. Szolnoki, A., Xie, N. G., Wang, C. \& Perc, M. Imitating emotions instead of strategies in spatial games elevates social welfare. EPL 96, 38002 (2011).

51. Szolnoki, A., Xie, N. G., Ye, Y. \& Perc, M. Evolution of emotions on networks leads to the evolution of cooperation in social dilemmas. Phys. Rev. E 87, 042805 (2013).

52. Smith, J. M. \& Parker, G. A. The logic of asymmetric contests. Anim. Behav. 24, 159-175 (1976).

53. Basil, M. Identification as a mediator of celebrity effects. J. Brodcast. Electron. 40, 478-495 (1996).

54. Jackson, D. \& Darrow, T. The influence of celebrity endorsements on young adults' political opinions. Int. J. Press-Polit. 10, 80-98 (2005).

55. Chen, X. J., Fu, F. \&Wang, L. Influence of different initial distributions on robust cooperation in scale-free networks: A comparative study. Phys. Lett. A 372, 1161-1167 (2008).

56. Ohtsuki, H., Hauert, C., Lieberman, E. \& Nowak, M. A. A simple rule for the evolution of cooperation on graphs and social networks. Nature 441, 502-505 (2006).

57. Nowak, M. A. Five rules for the evolution of cooperation. Science 314, 1560-1563 (2006).

58. Enquist, M. \& Leimar, O. The evolution of fatal fighting. Anim. Behav. 39, 1-9 (1990).

59. Szabó, G. \& Fáth, G. Evolutionary games on graphs. Phys. Rep. 446, 97-216 (2007).

60. Taylor, P. D. \& Jonker, L. B. Evolutionary stable strategies and game dynamics. Math. Biosci. 40, 145-156 (1978).

61. Zeeman, E. C. Global Theory of Dynamical Systems (Springer, 1980).

62. Wang, J., Wu, B., Wang, L. \& Fu, F. Consensus of population systems with community structures. Phys. Rev. E 78, 051923 (2008)

63. Girvan, M. \& Newman, M. Community structure in social and biological networks. Proc. Natl. Acad. Sci. USA 99, 7821-7826 (2002).

64. Newman, M. Modularity and community structure in networks. Proc. Natl. Acad. Sci. USA 103, 8577-8582 (2006).

65. Palla, G. Derenyi, I., Farkas, I. \& Vicsek, T. Uncovering the overlapping community structure of complex networks in nature and society. Nature 435, 814-818 (2005).

66. Onnela, J. P. Structure and tie strengths in mobile communication networks. Proc. Natl. Acad. Sci. USA 104, 7332-7336 (2007).

67. Lambiotte, R., Ausloos, M. \& Holyst, J. A. Majority model on a network with communities. Phys. Rev. E 75, 030101 (2007). 
68. Fu, F., Tarnita, C. E., Christakis, N. A., Wang, L., Rand, D. G. \& Nowak, M. A. Evolution of in-group favoritism. Sci. Rep. 2, 460 (2012).

\section{Acknowledgments}

The authors are supported by NSFC (61020106005 and 61375120).

\section{Author contributions}

K.L., R.C., T.W. and L.W. performed analyses, discussed the results, and contributed to the text of the manuscript.

\section{Additional information}

Competing financial interests: The authors declare no competing financial interests.

How to cite this article: Li, K., Cong, R., Wu, T. \& Wang, L. Bluffing promotes overconfidence on social networks. Sci. Rep. 4, 5491; DOI:10.1038/srep05491 (2014).

(c) (1) $\Theta$ This work is licensed under a Creative Commons Attribution-NonCommercialcc) this article are included in the article's Creative Commons license, unless indicated otherwise in the credit line; if the material is not included under the Creative Commons license, users will need to obtain permission from the license holder in order to reproduce the material. To view a copy of this license, visit http:// creativecommons.org/licenses/by-nc-nd/4.0/ 